\title{
Histiocitosis sinusal cutánea. Estudio clínico-patológico de 33 pacientes en el Centro Dermatológico Pascua
}

\author{
Cutaneous sinus histiocytosis. Clinicopathological study \\ of 33 patients at the Centro Dermatólogico Pascua
}

\author{
Gisela Navarrete Franco, ${ }^{*}$ José Alberto Ramos Garibay, ${ }^{\ddagger}$ Maribet González González, ${ }^{\S}$ \\ José Pablo Laguna Meraz," María Guadalupe Domínguez Ugalde**
}

\section{RESUMEN}

Introducción: La histiocitosis sinusal cutánea constituye una forma clínica de la enfermedad de Rosai-Dorfman o histiocitosis sinusal con linfadenopatía masiva (HSLM). Esta variedad limitada a piel se considera poco frecuente. Objetivo: Conocer la incidencia, epidemiología y características clínico-patológicas de esta entidad en el Centro Dermatológico "Dr. Ladislao de la Pascua» (CDP). Material y métodos: Se realizó un estudio retrospectivo, transversal, descriptivo y observacional con los archivos del Servicio de Dermatopatología del CDP en un periodo de 34 años (1985-2019) y se seleccionaron los casos con diagnóstico histológico de histiocitosis sinusal y por correlación clínica, con manifestaciones únicamente cutáneas. Resultados: Se encontraron en total 33 casos, predominó el género femenino con 24 mujeres. La edad fluctuó entre nueve y 81 años, con una media de 36 años. La topografía más frecuente fue en la cabeza, la morfología muy diversa; la evolución crónica y en su mayoría asintomática. Histológicamente, el cuadro fue muy característico, se observó emperipolesis en todos los casos. Conclusiones: La histiocitosis sinusal cutánea (HSC) no es tan rara como se pensaba, consideramos que su incremento en estos últimos años se debe al mayor conocimiento tanto clínico como histológico de la entidad, de ahí nuestro interés de comunicar esta casuística.

Palabras clave: Histiocitosis sinusal cutánea, emperipolesis, enfermedad de Rosai-Dorfman.

\section{ABSTRACT}

Introduction: Cutaneous sinus histiocytosis (CSH) is a clinical form of Rosai-Dorfman disease or sinus histiocytosis with massive lymphadenopathy (SHLM), this variety limited to the skin is considered rare. Objective: To know the epidemiology and clinical-pathological characteristics of this entity in the Centro Dermatológico «Dr. Ladislao de la Pascua" (CDP) in Mexico City. Material and methods: A retrospective, cross-sectional, descriptive and observational study was performed with the records of the CDP Dermatopathology department in 34 years (19852019). Cases with histological diagnosis of sinus histiocytosis and only cutaneous disease (by clinical data) where selected. Results: A total of 33 cases were found, the female gender predominated in 24; the age was between nine and 90 years, with a median of 36 . The most frequent location was the face, the clinical aspect was diverse; most cases were chronic and with an asymptomatic evolution. Histologically, the image was characteristic, emperipolesis was observed in all cases. Conclusions: Cutaneous sinus histiocytosis (CSH) is not as rare as was previously thought, we consider that its recently increase in diagnosis relies on greater clinical and histological knowledge of this disease, hence our interest in communicating this series of cases.

Keywords: Cutaneous sinus histiocytosis, emperipolesis, Rosai-Dorfman disease.

\footnotetext{
* Jefe del Servicio de Dermatopatología.

* Subjefe del Servicio de Dermatopatología.

$\S$ Dermatopatóloga adscrita.

" Residente de segundo año de Dermatopatología.

** Dermatóloga y Dermatopatóloga.

Citar como: Navarrete FG, Ramos GJA, González GM, Laguna MJP, Dominguez UMG. Histiocitosis sinusal cutánea. Estudio clínico-patológico de 33 pacientes en el Centro Dermatológico Pascua. Rev Cent Dermatol Pascua. 2021; 30 (2): 61-67. https://dx.doi.org/10.35366/101175 


\section{INTRODUCCIÓN}

La histiocitosis sinusal con linfadenopatía masiva (HSLM) o enfermedad de Rosai-Dorfman es una entidad poco frecuente, de causa desconocida y caracterizada por linfadenopatía cervical, fiebre, leucocitosis, velocidad de sedimentación acelerada e hipergammaglobulinemia; todo esto asociado a un cuadro histológico característico. ${ }^{1}$

Puede afectar otros órganos como ojos, tracto respiratorio, testículos, hueso y piel..$^{1,2}$

Destombes, en 1965, fue el primero en llamar la atención sobre esta patología, al publicar cuatro casos con el nombre de Adenitis con sobrecarga lipídica del niño o del adulto joven, observados en las Antillas y Mali, señalando todos los hallazgos clínico-patológicos que más tarde, en 1969, describieron Rosai y Dorfman con el nombre de «histiocitosis sinusal con linfadenopatía masiva».3

En 1978, Thawerani y colaboradores publicaron por primera vez las manifestaciones cutáneas de esta entidad, señalando el mismo cuadro histológico que en los ganglios. ${ }^{4}$

Epidemiológicamente, la HSLM afecta todas las razas, especialmente la negra, con predilección en niños $y$ jóvenes de sexo masculino. ${ }^{1-4}$

\section{MATERIAL Y MÉTODOS}

Se realizó un estudio retrospectivo, transversal, descriptivo y observacional.

Se revisó el material del Servicio de Dermatopatología del Centro Dermatológico «Dr. Ladislao de la Pascua» de la Ciudad de México, en un lapso de 34 años (1985-2019).

Se tomaron los datos epidemiológicos y clínicos de las solicitudes de los estudios histopatológicos y se revisaron las laminillas correspondientes.

Todas las piezas fueron trabajadas por el método de parafina y teñidas con tinción de rutina (H\&E), así como proteína S100.

\section{RESULTADOS}

Los resultados finales se resumen en la Tabla 1. Se encontró un total de 33 casos, de los cuales 24 correspondieron al sexo femenino y nueve al masculino. Con respecto a la edad, el menor fue de nueve años y el mayor de 81, existiendo dos picos: la tercera y quinta décadas de la vida.

Clínicamente, la topografía fue localizada en 25 casos y de éstos, 12 sólo en la cabeza, siguiendo el tronco con seis, extremidades inferiores con tres, dos en cuello y dos en extremidades superiores. La dermatosis fue diseminada en ocho pacientes.

Morfológicamente: 21 mostraron nódulos, nueve placas y tres morfología combinada (nódulos y placas).

El tiempo de evolución fue de dos meses a cinco años, consultando 18 de ellos en el primer año de la dermatosis.

Los diagnósticos clínicos enviados fueron de tumores en 24 casos, inflamatorias cinco, HSC en dos y dos en estudio.

Histopatológicamente, la epidermis presentó atrofia en 19 casos, acantosis en 10, ulceración en uno y tres sin alteraciones. Otros cambios epidérmicos: paraqueratosis en seis casos e hiperpigmentación de la capa basal en dos. Se observó en 16 la presencia de una banda subepidérmica de tejido colágeno normal (zona de Grenz).

Los cambios importantes se localizaron principalmente en toda la dermis en 15 casos e invadiendo tejido celular subcutáneo en 18.

El infiltrado estuvo constituido en todos los casos por acúmulos de células histiocíticas, grandes, de citoplasma eosinófilo, abundante y pálido, algunas de las cuales contenían células inflamatorias como linfocitos, células plasmáticas y neutrófilos, fenómeno que se conoce con el nombre de «emperipolesis», mismo que se presentó en todos los casos, variando únicamente el grado de intensidad.

Por otro lado, estas células histiocíticas se disponían en mantos en algunas áreas y en otras se entremezclaban con neutrófilos, los cuales se presentan generalmente formando microabscesos; asimismo, como parte del infiltrado, se observaron abundantes células plasmáticas, linfocitos y eosinófilos.

Los cuerpos de Russell estuvieron presentes en todos los casos, la formación de centros germinales (folículos linfoides) en 14 y fibrosis en 12.

Los cambios vasculares constantes en todos los casos fueron: intensa dilatación, congestión y engrosamiento de paredes vasculares.

La proteína S100 fue positiva en todos los casos.

\section{DISCUSIÓN Y COMENTARIOS}

La histiocitosis sinusal con linfadenopatía masiva, se considera, desde 1972, como una entidad clínico-patológica. ${ }^{2}$ Desde entonces, se ha asociado el involucro de otros órganos, de los cuales se manifiesta en la piel en $43 \%$, con participación del tejido linfático; sin embargo, la histiocitosis sinusal limitada exclusivamente a la piel, 
que también se conoce como puramente cutánea, se observa en $3 \%$, por lo que se considera poco frecuente. ${ }^{5}$ Existen comunicaciones de casos aislados de la forma puramente cutánea ${ }^{6-20}$ y pocas series de casos..$^{21-26}$

La enfermedad de Rosai-Dorfman fue clasificada en 1987 por el grupo de trabajo de la Sociedad del Histiocito como una histiocitosis de células No Langerhans, y posteriormente la reclasifican de acuerdo con características clínicas, radiográficas, patológicas, fenotípicas, genéticas y moleculares, separando a la histiocitosis sinusal clásica ganglionar de la puramente cutánea, ${ }^{27}$ haciendo ver que el concepto de trastorno histiocítico reactivo, que carecía de clonalidad, está cambiando al menos en un subgrupo de casos donde parece evidente una naturaleza clonal, ya que se han descrito mutaciones de quinasa en la histiocitosis sinusal ganglionar y extraganglionar, pero no en la puramente cutánea, donde no se pudo encontrar datos sobre el perfil molecular, lo que hace suponer que la enfermedad de Rosai-Dorfman clásica (ganglionar y extraganglionar) es una entidad diferente a la puramente cutánea. ${ }^{27,28}$

En nuestro trabajo, encontramos que desde el punto de vista epidemiológico: los 33 pacientes son de raza mestiza, el sexo correspondió al femenino de manera predominante y la edad mayormente observada fue en adultos de la tercera y sexta décadas de la vida, lo que difiere de la HSLM.

Por otro lado, clínicamente la topografía fue muy variable, ya que tuvimos oportunidad de observar las lesiones en todos los segmentos, predominando en cara y tronco.

Morfológicamente vimos gran diversidad de lesiones: nódulos, en número y tamaño variable desde unos cuantos milímetros hasta lesiones de aspecto tumoral con presencia de telangiectasias en su superficie; otros más que confluían para formar placas de número, forma y tamaño variable; mientras que en otros más: combinación de nódulos aislados en unas zonas y en otras placas con presencia de nódulos satélites.

Algo que nos parece importante destacar es la presencia de lesiones en diferentes fases de desarrollo en un mismo paciente, sobre todo en aquellos cuya topografía estaba diseminada a varios segmentos y la morfología era diversa, incluso en una misma lesión se pudo observar actividad e involución de la misma, lo cual nos habla de lo autolimitado del proceso y el buen pronóstico del mismo.

Nuestros pacientes no presentaron alteraciones de tipo sistémico ni de laboratorio, exclusivamente lesiones confinadas a piel.

Con respecto al seguimiento de los casos, la mayoría mostró tendencia a la involución de la dermatosis. En los que presentaron lesiones únicas se realizó extirpación de las mismas.

Con estas observaciones y las descritas en la literatura, nosotros consideramos que la HSC comparte características histopatológicas con la forma clásica ganglionar de la enfermedad, mas no las epidemiológicas ni clínicas como ya se hace ver en estudios previos. ${ }^{5,23,28}$ La forma puramente cutánea presenta identidad propia, que la ubicaría en un sitio extremo del espectro de manifestaciones diversas, como sería la de involucro ganglionar en el intermedio y en el otro extremo del espectro, la forma extraganglionar o sistémica que afecta otros órganos como hueso (10-20\%), tracto respiratorio (20\%), sistema nervioso central (10\%), entre otros, ${ }^{29}$ lo que explicaría la amplia gama de datos clínicos, teniendo el mismo sustrato histopatológico. 
Tabla 1: Datos clínicos.

\begin{tabular}{|c|c|c|c|c|c|c|}
\hline Paciente & Sexo & Edad & Topografía & Morfología & Evolución & Dx. clínico \\
\hline 1 & $\mathrm{~F}$ & 58 & Cabeza (cara) & Nódulo 4 cm & 5 meses & CBC vs Linfoma \\
\hline 2 & $\mathrm{~F}$ & 29 & Muslo & Placa eritematoviolácea $8 \mathrm{~cm}$ & 8 meses & Hemangiolinfangioma \\
\hline 3 & $\mathrm{~F}$ & 19 & Tronco (mama) & Placa eritematoinfiltrada $7 \mathrm{~cm}$ & 1 año & D. crónica \\
\hline 4 & $\mathrm{~F}$ & 28 & Tronco (hemitórax) & Exofítica $1 \mathrm{~cm}$ & 14 meses & Pilomatrixoma \\
\hline 5 & $\mathrm{~F}$ & 53 & Cabeza (cara) & Placas $1-3 \mathrm{~cm}$ & 3 meses & Lupus vs Tb luposa \\
\hline 6 & $\mathrm{~F}$ & 29 & Brazo & Nódulos y placa $8 \mathrm{~cm}$ & 6 meses & Piloleiomioma \\
\hline 7 & M & 49 & Cabeza, tronco y extremidad & Nódulos & 18 meses & En estudio \\
\hline 8 & $\mathrm{~F}$ & 9 & Muslo & Nódulo $1 \mathrm{~cm}$ & 1 año & Tumor de anexos \\
\hline 9 & $\mathrm{~F}$ & 53 & Cabeza, tronco y extremidad & Nódulos y placas & 3 años & Histiocitosis sinusal \\
\hline 10 & $\mathrm{~F}$ & 81 & Cuello & Nódulo 4 cm & 2 meses & Cicatriz queloide \\
\hline 11 & $\mathrm{~F}$ & 79 & Cabeza y cuello & Nódulo $0.3 \mathrm{~cm}$ & 2 meses & Espiradenomas \\
\hline 12 & M & 57 & Cabeza (cara) & Placas $5 \mathrm{~cm}$ & 2 meses & Sarcoma de Kaposi \\
\hline 13 & $\mathrm{~F}$ & 43 & Cabeza (cara) y cuello & Placas $1-6 \mathrm{~cm}$ & 8 meses & Histiocitosis sinusal \\
\hline 14 & $\mathrm{~F}$ & 20 & Cuello & Nódulo $0.5 \mathrm{~cm}$ & 6 meses & Angioma \\
\hline 15 & $\mathrm{~F}$ & 52 & Pabellón auricular & Placa & 8 meses & En estudio \\
\hline 16 & $\mathrm{~F}$ & 26 & Tronco (abdomen) & Placa $3.5 \mathrm{~cm}$ & 1 año & Linfangioma \\
\hline 17 & $\mathrm{~F}$ & 48 & Abdomen y extremidades & Placas y nódulos & 3 años & Prúrigo nodular \\
\hline 18 & $\mathrm{~F}$ & 18 & Tórax y hombro & Nódulo violáceo y doloroso & 2 meses & Quiste epidérmico \\
\hline 19 & $\mathrm{~F}$ & 35 & $\begin{array}{l}\text { Miembro inferior } \\
\text { (muslo y región poplítea) }\end{array}$ & Nódulo subcutáneo & 1 año & Sarcoma \\
\hline 20 & $\mathrm{~F}$ & 36 & $\begin{array}{l}\text { Cabeza (cara: surco } \\
\text { nasogeniano) }\end{array}$ & Nódulo $1.2 \mathrm{~cm}$ & 1 año & Neurofibroma \\
\hline 21 & $\mathrm{~F}$ & 29 & $\begin{array}{l}\text { Cabeza } \\
\text { (cara: mejillas y región malar) }\end{array}$ & Nódulos c/telangiectasias & 1 año & $\begin{array}{l}\text { Enfermedad } \\
\text { granulomatosa }\end{array}$ \\
\hline 22 & $\mathrm{~F}$ & 27 & Cabeza (cara: región malar) & Nódulos (9) $0.2-0.5 \mathrm{~cm}$ & 5 meses & Sarcoidosis \\
\hline 23 & M & 13 & Cabeza (cara) & Nódulo subcutáneo amarillento & 1 año & Lipoma \\
\hline 24 & $\mathrm{~F}$ & 18 & Brazo & Nódulo subcutáneo, indurado & 2 años & Fibrolipoma \\
\hline 25 & M & 33 & Tronco (tórax y abdomen) & Placa infiltrada c/nódulos & 2 años & Dermatofibroma, DFSP \\
\hline 26 & $\mathrm{~F}$ & 26 & Cabeza (cara: frente) y tronco & Nódulos c/telangiectasias & 5 meses & Xantogranuloma \\
\hline 27 & M & 61 & $\begin{array}{l}\text { Cabeza, tronco y miembros } \\
\text { inferiores }\end{array}$ & Nódulos eritematoinfiltrados & 6 meses & Histiocitosis sinusal \\
\hline 28 & M & 24 & Cabeza (piel cabelluda) & Nódulo $3 \mathrm{~cm}$ c/ telangiectasias & 5 años & Condroma \\
\hline 29 & $\mathrm{~F}$ & 22 & Tronco (tórax anterior) & Placa $4 \mathrm{~cm}$ eritematosa & 6 meses & Sarcoma de Kaposi \\
\hline 30 & $\mathrm{~F}$ & 18 & $\begin{array}{l}\text { Tronco } \\
\text { (tórax: región supraclavicular) }\end{array}$ & Nódulo $0.8 \times 0.3 \mathrm{~cm}$ eritematosos & 5 meses & $\begin{array}{l}\text { Dermatofibroma vs } \\
\text { Linfocitoma }\end{array}$ \\
\hline 31 & M & 15 & Cabeza (piel cabelluda y cara) & Nódulo subcutáneo $2 \mathrm{~cm}$ & 18 meses & Tumor de anexos \\
\hline 32 & M & 72 & Cabeza (región retroauricular) & Nódulo subcutáneo $1 \mathrm{~cm}$ & 11 meses & Tumor de anexos \\
\hline 33 & M & 28 & Cabeza (pabellón auricular) & Nódulo $5 \mathrm{~cm}$ eritematoso & 5 meses & Tumor de anexos \\
\hline
\end{tabular}


Figura 1: Aspectos clínicos de la histiocitosis sinusal cutánea.
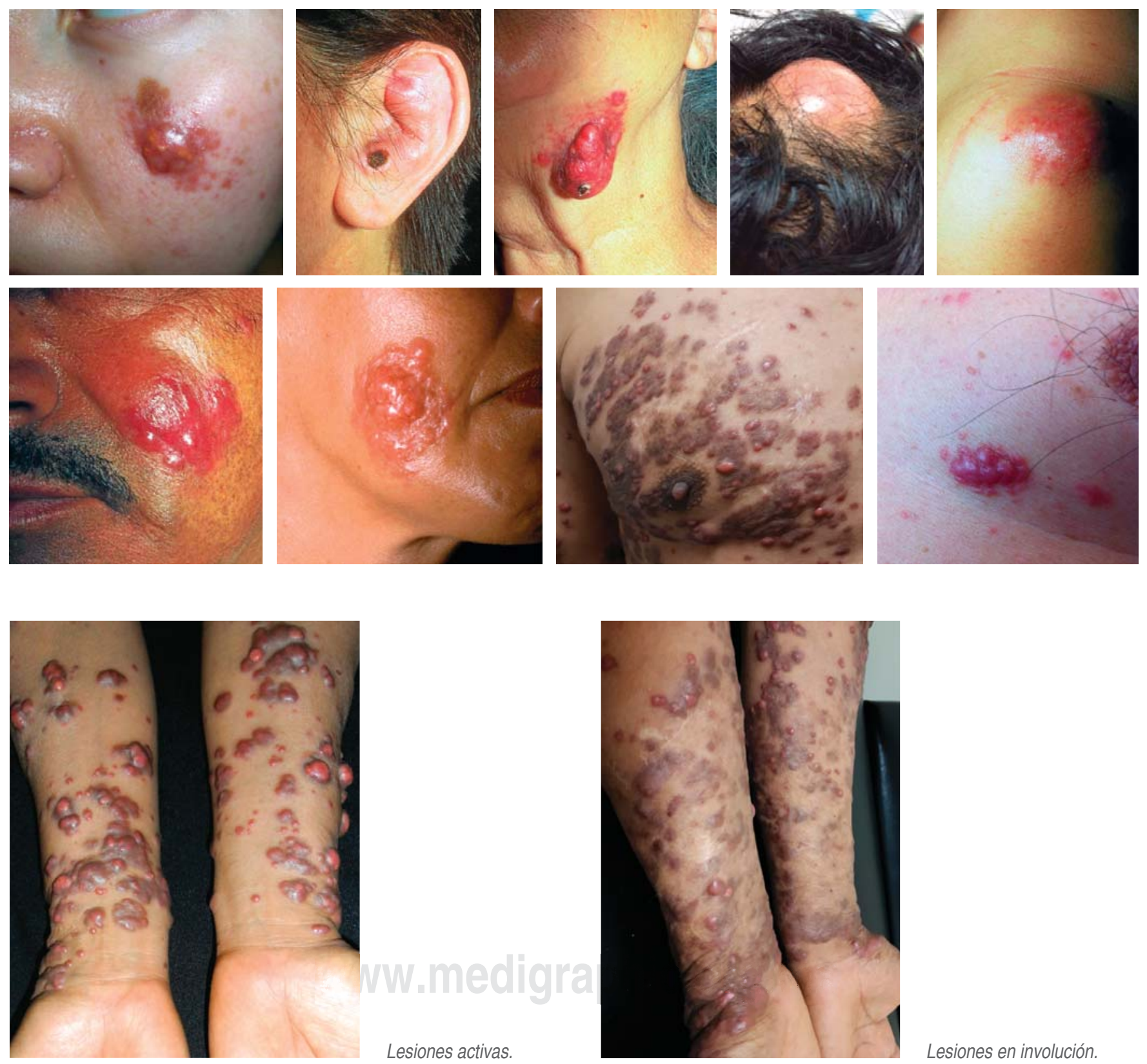

Lesiones activas.

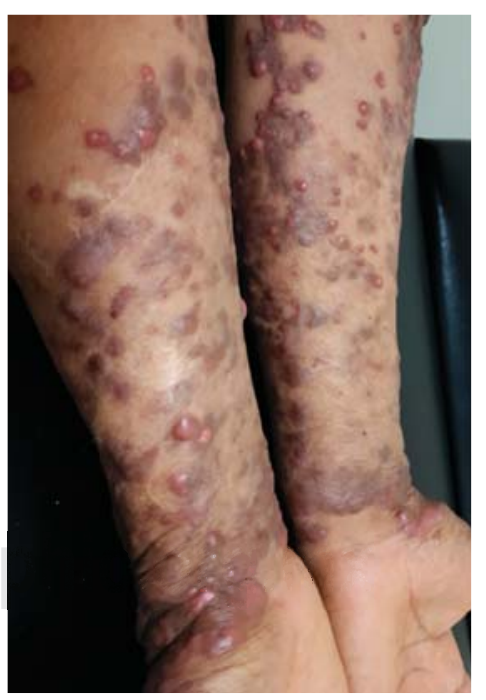

Lesiones en involución. 


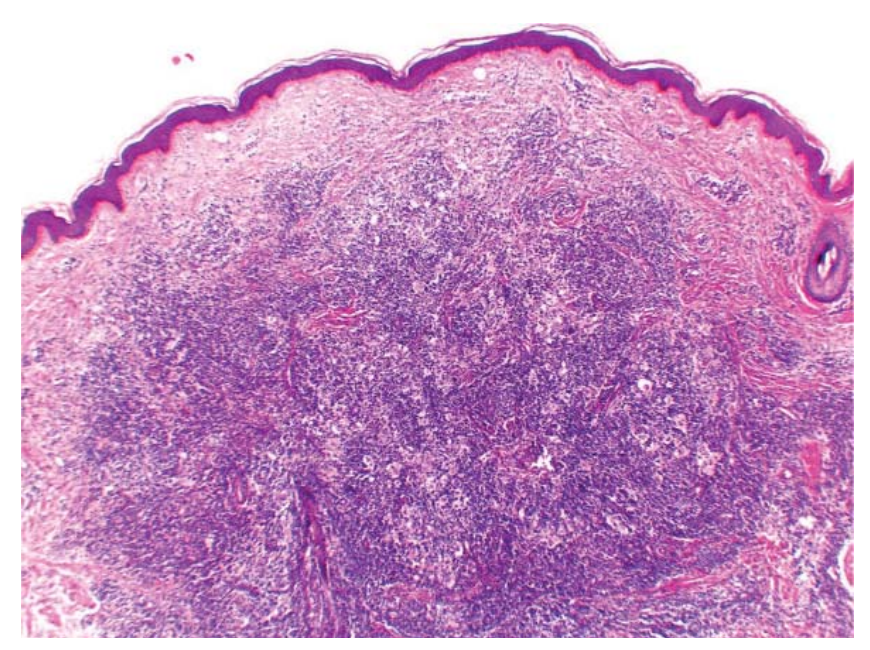

Figura 2: Histiocitosis sinusal (H\&E 4x).

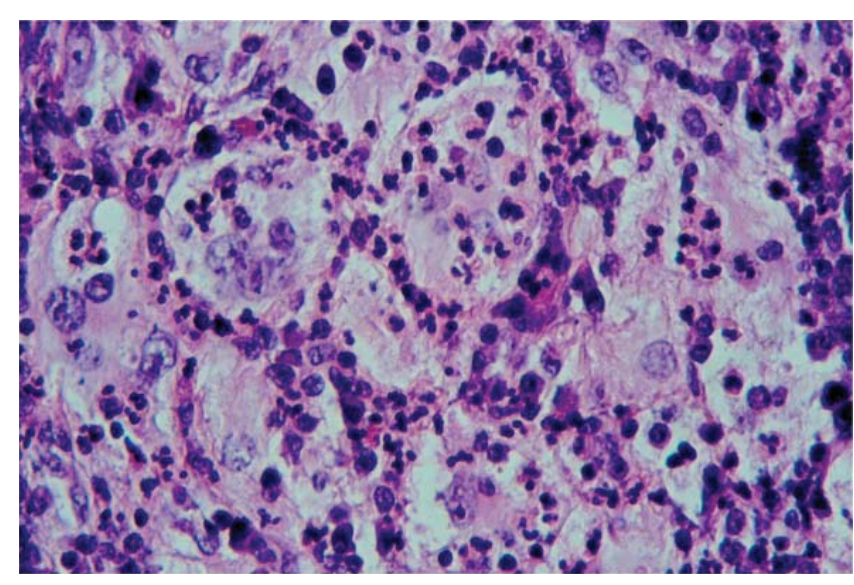

Figura 3: Histiocitosis sinusal. Emperipolesis (H\&E 40x).

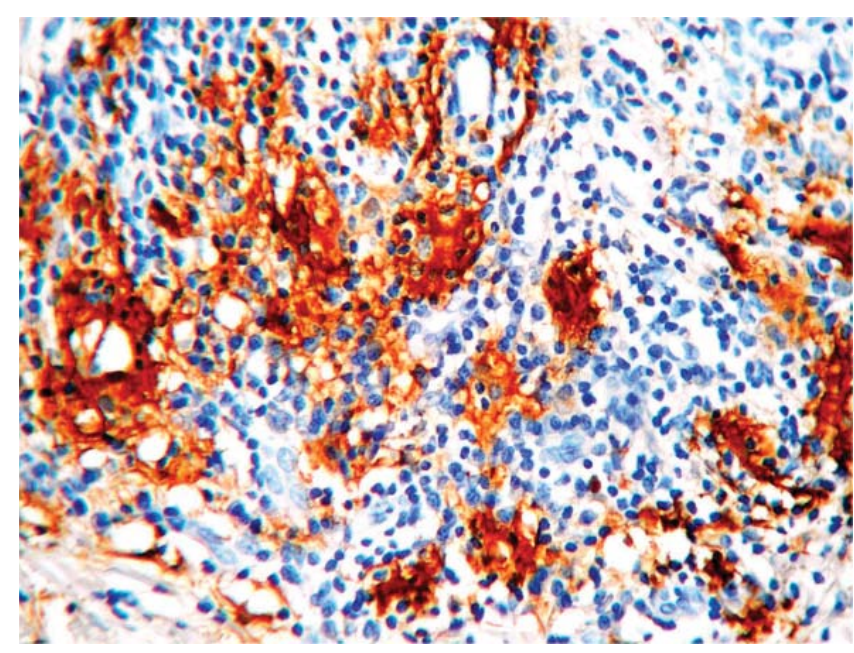

Figura 4: Inmunohistoquímica (proteína S-100).

\section{REFERENCIAS}

1. Rosai J, Dorfman RF. Sinus histiocytosis with massive lymphadenopathy. A newly recognized benign clinic-pathological entity. Arch Pathol. 1969; 87: 63-70.

2. Rosai J, Dorfman RF. Sinus histiocytosis with massive limphadenopathy: a pseudolinfomatous benign disorder. Analysis of 34 cases. Cancer. 1972; 30: 1174-1188.

3. Destombes P. Adénites avec surcharge lipidique de l'enfant au de l'adulte jeune, observées aux Antilles et au Mali (quatre observations). Bull Soc Pathol Exot Filiales. 1965; 58: 1169-1175.

4. Thawerani N, Sanchez RL, Rosai J, Dorfman RF. The cutaneous manifestations of sinus histiocytosis with massive limphadenopathy. Arch Dermatol. 1978; 114: 191-197.

5. Gaul M, Chang T. Cutaneous Rosai-Dorfmann disease. Cutis. 2019; 103: 171-173.

6. Lazar AP, Esterly NB, González-Cruzi F. Sinus histiocytosis clinically limited to the skin. Pediatric Dermatol. 1987; 4: 247-253.

7. Mac-Moune LF, Lam WY, Chin CW, Ng WL. Cutaneous Rosai-Dorfman disease presenting as a suspicious breast mass. J Cutan Pathol. 1994; 21: 377-382.

8. Pérez A, Rodríguez M, Febrer I, Aliaga A. Sinus histiocytosis confined to the skin. Case report and review of the literature. Am J Dermatopathol. 1995; 17: 384-388.

9. Moon KJ, Ick YW, Min KS, Lee M. Sinus Histiocytosis (Rosai-Dorfman Disease) Clinically Limited to the Skin. Acta Derm Venereol. 1999; 79: 363-365.

10. Kroumpouzos G, Demierre MF. Cutaneous Rosai-Dorfman disease: histopathological presentation as inflammatory pseudotumor. A literature review. Acta Derm Venereol. 2002; 82: 292-296.

11. Kenneth KK, Yoke TP, Thamboo TP. Bilateral cauliflower ear deformity: an unusual presentation of cutaneous Rosai-Dorfman disease. Plast Reconstr Surg. 2004; 13: 967-969.

12. Beltrán G, Navarro F, Meza B, Bravo F, Santos R. Enfermedad de Rosai-Dorfman Cutánea: Tratamiento con nitrógeno líquido. Dermatol Perú. 2004; 14: 200-203.

13. González-Conde E, Domínguez-Malagón H, Cano-Valdez AM. Enfermedad de Rosai-Dorfman cutánea. Patología. 2005;43(1):42-43.

14. Chan CC, Chu CY. Dapsone as a potential treatment for cutaneous Rosai-Dorfman disease with neutrophilic predominance. Arch Dermatol. 2006; 142: 428-430.

15. Zaragoza NV, Martínez CL, Alegre de Miguel V, Vilata CJJ. Enfermedad de Rosai-Dorfman cutánea. Piel. 2008; 23(5): 227-9.

16. Quispetira J, Moisés C, Paredes A, Sánchez G, Pacheco M, Carbajal T. Enfermedad de Rosai-Dorfman cutánea: reporte de caso y revisión de la literatura. Folia dermatol. Peru 2009; 20: 91-94.

17. Cook-Norris, Hayes BB, Robb C, Boyd AS, Zic JA. Purely cutaneous Rosai-Dorfman disease. Int J Dermatol. 2009; 48: 439-440.

18. Cabrera HN, Fernández VMS, Verdejo G, Casas JG. Enfermedad de Rosai-Dorfman: forma clínica acneiforme. Dermatol Argent. 2010; 16 : 23-26.

19. Fung THT. Cutaneous Rosai-Dorfman disease presenting as a solitary thigh mass. Hong Kong J Orthop. 2010; 14: 13-15.

20. Fumerton R, Ball N, Zhou Y. Refractary cutaneous Rosai-Dorfman disease responsive to cryotherapy. Cutis. 2011; 87: 296-299. 
21. Navarrete G, Novales J, Pinto D, Martínez C, Ramos-Garibay A. Histiocitosis sinusal cutánea (enfermedad de Rosai-Dorfman). Comunicación de cuatro casos. Med Cutan Iber La Am. 1996; 24:39-43.

22. Navarrete FG, Novales J, Jurado SCF, Medina CD, Lopeztello SL, Mendoza AL. Histiocitosis sinusal cutánea. Estudio clínico-patológico. Dermatol Rev Mex. 2001; 45(4): 173-179.

23. Brenn T, Calonje E, Granter SR, Leonard N, Grayson W, Fletcher CD, McKee PH. Cutaneous Rosai-Dorfman disease is a distinct clinical entity. Am J Dermatopathol. 2002; 24: 385-391.

24. Ortiz-Hidalgo C, Cuesta-Mejías T, Ochoa-Ochoa C, ValenzuelaEspinosa A, Toussaint-Caire S. Enfermedad de Rosai-Dorfman, limitada a la piel. Informe de cuatro casos. Gac Med Mex. 2003; 139: 1-6.

25. Wang KH, Chen WY, Liu HN, Huang CC, Lee WR, Hu CH. Cutaneous Rosai-Dorfman disease: clinicopathological profiles, spectrum and evolution of 21 lesions in six patients. Br J Dermatol. 2006; 154:277-286.

26. Kong YY, Kong JC, Shi DR, Lu HF, Zhu XZ, Wang J et al. Cutaneous Rosai-Dorfman Disease: a clinical and histopathologic study of 25 cases in China. Am J Surg Pathol. 2007; 31: 341-350.
27. Emile JF, Abla O, Fraitag S, Horne A, Harocle J, Donadieu J et al. Revised classification of histiocytoses and neoplasm of the mecrophage-dendritic cell lineages. Blood. 2016; 127: 2672-2681.

28. Bruce-Brand C, Schneider JW, Schubert P. Rosai-Dorfman disease: an overview. J Clin Pathol. 2020; 73: 697-705.

29. Goyal G, Young JR, Koster MJ, Tobin WO, Vassallo R, Ryu JH et al. The Mayo Clinic Histiocytosis Working Group Consensus Statement for the Diagnosis and Evaluation of Adult Patients with Histiocytic Neoplasms: Erdheim-Chester Disease, Langerhans Cell Histiocytocis and Rosai-Dorfman Disease. Mayo Clin Proc. 2019; 94: 2054-2071.

Correspondencia:

Gisela Navarrete Franco

E-mail: giselanavarrete@ hotmail.com 